\title{
Transatlantica
}

Revue d'études américaines. American Studies Journal

\section{Pierre-Marie Loizeau, Martin Van Buren : The Little Magician, Hauppauge, NY, Nova History Publ., 2009}

Jean-Marc Serme

\section{OpenEdition}

1 Journals

Édition électronique

URL : https://journals.openedition.org/transatlantica/4857

DOI : $10.4000 /$ transatlantica.4857

ISSN : 1765-2766

Éditeur

Association française d'Etudes Américaines (AFEA)

Référence électronique

Jean-Marc Serme, «Pierre-Marie Loizeau, Martin Van Buren : The Little Magician, Hauppauge, NY, Nova History Publ., 2009 », Transatlantica [En ligne], 1 | 2010, mis en ligne le 27 septembre 2010, consulté le 10 février 2023. URL : http://journals.openedition.org/transatlantica/4857 ; DOI : https://doi.org/

10.4000/transatlantica.4857

Ce document a été généré automatiquement le 10 février 2023.

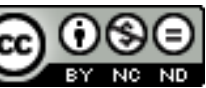

Creative Commons - Attribution - Pas d'Utilisation Commerciale - Pas de Modification 4.0 International - CC BY-NC-ND 4.0

https://creativecommons.org/licenses/by-nc-nd/4.0/ 


\section{Pierre-Marie Loizeau, Martin Van Buren: The Little Magician, Hauppauge, NY, Nova History Publ., 2009}

Jean-Marc Serme

1 La biographie de Martin Van Buren par Pierre-Marie Loizeau trouve sa place parmi un nombre non négligeable d'ouvrages en anglais consacrés ces dernières années au huitième président des États-Unis. Ce grand politique d'avant la guerre de Sécession fut méprisé pendant longtemps par les historiens. Il faut dire qu'il paraissait bien frêle entre les deux imposants « noyers » d'Amérique : le Vieux, Andrew Jackson, et le Jeune, James K. Polk. Outre sa réputation de Renard Rusé, son goût immodéré pour le compromis ne lui valut pendant longtemps qu'un grand mépris, qui en fit l'antithèse des héros belliqueux et intransigeant que l'on adore adorer. Pourtant, Van Buren est crédité de la formation du système bipartite américain que l'on connaît aujourd'hui, et surtout de la naissance du Parti démocrate. Il est donc reconnu pour son excellence en matière institutionnelle et organisationnelle, ce sur quoi insistent les monographies récentes.

2 Au vu du corpus américain consacré depuis les années 1970 à Van Buren, Loizeau aurait pu choisir de servir sa biographie en français. L'entreprise toutefois est louable dans son intention de réhabiliter un politicien « rusé mais avec des principes » (117), un de ces hommes de la pénombre qui apparaît plus complexe et plus intéressant à mesure qu'on apprend à le connaître. Toutefois, on pourra regretter le ton académique de cette biographie qui aurait pu bénéficier d'une narration moins conventionnelle. Mais c'est peut-être le cadre dans lequel s'insère le livre qui veut cela. Le but de cette collection, First Men, America's Presidents Series, est de valoriser l'action de ces hommes d'exception que furent les présidents, censés représenter " la sagesse, le leadership et l'intégrité » et de souligner leur caractère unique, dans un schéma narratif défini à l'avance par les éditeurs. Et ainsi que l'explique la responsable de publication: « The two major themes of 
this series are the character traits marking success in the presidency, and the changes in the office of the presidency through America's history. Character matters in all walks of life, but perhaps matters most within the character of the President of the United States. "(xi) Tout cela peut paraitre un peu daté, à la fois dans l'approche et la formulation, ce qui peut expliquer le conformisme formel et historique de l'ouvrage.

Martin Van Buren est le premier président «hollandais » de l'histoire (les Roosevelt, Theodore, Franklin et Eleonore étaient également de lointains descendants des premiers colons hollandais de New York - ancienne Nouvelle Amsterdam). Il a participé au mouvement de réforme politique de son État dans les années 1810 avant de rejoindre le Congrès puis le Cabinet du président Jackson en 1828. C'était, dans la lignée des anti-fédéralistes jeffersoniens, un âpre défenseur du droit des États. Lors de la Panique de 1837, il refusa d'apporter le soutien de l'État fédéral à une nation plongée dans l'une des premières graves récessions qui ont ponctué l'histoire économique et sociale du XIX ${ }^{\mathrm{e}}$ siècle. Mais il soutiendra à la fin de sa vie la guerre d'Abraham Lincoln pour préserver l'intégrité de l'Union américaine (182).

4 A l'instar des historiens contemporains qui se sont penchés à nouveau sur Van Buren depuis le début des années 1980, Loizeau réévalue très largement sa participation active aux profondes transformations des partis politiques américains dans la première partie du siècle. Il rappelle aussi longuement et avec raison qu'il fut un des architectes avisés de l'alliance du Parti démocrate de New York avec les sudistes de Virginie et de Caroline du Sud, que Van Buren appelle ses «affiliations sudistes». C'est un aspect important dans une période de grande tension entre les régions, après une première alerte en 1832 lors de la Crise de la Nullification. L'invalidation par la Caroline du Sud emmenée par le vice président Calhoun de la loi sur les droits douaniers puis de la loi permettant au président d'imposer la législation fédérale aux États récalcitrants mena le pays au bord de la guerre civile. Finalement conciliant avec les « rebelles » et leurs détracteurs, le président Jackson déploya cependant toute la rhétorique nationaliste dont il était capable malgré son soutien aux thèses des droits des États. Lors d'un toast du 4 juillet, Van Buren tenta de concilier le droit des États et l'Union de ces derniers en appelant à des "concessions réciproques " (98), une position difficile à tenir dans le débat toujours plus virulent qui opposait le Nord au Sud. Van Buren conserva durant les mandats Jackson cet objectif d'alliance nationale au sein du Parti démocrate qu'il ne parvint pourtant pas à maintenir lors de sa propre présidence. Loizeau décrit efficacement cette position médiane difficile à tenir parmi les « passions montantes, les débats houleux et les impitoyables altercations » de la période (134).

Et c'est bien le paradoxe de cet homme qui transparaît dans cette biographie. Aussi rusé, habile, manipulateur qu'il fût, sa présidence en désenchanta beaucoup. Il s'était fait beaucoup d'ennemis en accédant à la Maison Blanche, au sein même du parti. D'ailleurs, Loizeau montre bien que le Parti démocrate était une structure fragile et minée par les dissensions internes (92-93). En outre, il hérita dès son accession, d'une crise économique majeure qu'il choisit de ne pas traiter, fidèle à ses principes de laissez-faire économique. Loizeau ici tente de défendre la cause difficile d'un président donnant des réceptions somptueuses dans un palais rénové alors que le pays comptait à la fin de 1837 plus de $30 \%$ de travailleurs sans emploi (et sans aucun soutien financier, il va sans dire). Ses ennemis politiques eurent beau recourir à de "grotesques" tirades contre lui, il n'en apparaît pas moins que Van Buren resta sourd aux affres de son pays (malgré quelques timides mesures sans effet); il fut sur ce point un ancêtre politique de 
Herbert Hoover, sujet comme lui aux incessantes moqueries d'une presse déchaînée et d'un peuple désespéré. Loizeau décrit habilement tout au long de son ouvrage la férocité des oppositions, qu'elles vinssent de la presse ou des membres du Congrès (146-150) et ce ressort permet de prendre la mesure d'un âge politique en pleine effervescence pour qui la vindicte publique relevait aussi d'une égalité politique nouvellement acquise.

Van Buren est le modèle du nouveau politicien de l'après-dynastie virginienne, un homme d'appareil aux convictions profondes mais aux stratégies multiples, aux alliances fluctuantes, aux amitiés intéressées; il est aussi atypique dans son désir de fédérer les énergies, voire les contraires (il accorda par exemple son soutien au Fédéraliste Rufus King). En cela, il est une sorte de Henry Clay Démocrate, mais qui aurait accédé à la magistrature suprême. Loizeau donne une définition plutôt pragmatique du personnage: "Martin avait cette rare capacité d'intuition, très utile en politique, de suivre le vent, de naviguer entre les différents courants et opinions tout en maintenant le cap, d'anticiper les dangers pour mieux les éviter, et de maintenir de franches amitiés tout du long. " Bien sûr, les hommes politiques des générations précédentes connaissaient eux aussi les règles de navigation en eaux troubles que requiert la gestion de la chose publique. Mais Van Buren et la nouvelle génération de leaders nés après la Déclaration d'Indépendance durent s'adapter, durant la période appelée « Démocratie jacksonienne ", à des changements fondamentaux de la société américaine que Loizeau rappelle en d'efficaces résumés historiques (121-122).

7 La peinture de l'époque qu'il donne sonne juste, bien que Van Buren y occupe une place très prépondérante, parfois au détriment de la diversité politique d'alors. On comprend que le but de l'auteur est de redorer le blason d'un «leader national que beaucoup ont oublié » (5), mais on aurait espéré un plus juste équilibrage des forces en présence, et notamment une prise en compte des différents groupes qui travaillèrent à l'élaboration du Parti démocrate durant la deuxième campagne présidentielle d'Andrew Jackson, car Martin Van Buren n'en fut pas le seul artisan. De même, Loizeau a tendance à qualifier certaines figures un peu rapidement, Burr est le «wily New Yorker» et Jackson demeure "impetuous» ou "indomitable » (107), les adjectifs convenus et réducteurs pour décrire un old Hickory qui, sauf à apparaître comme la figure légèrement stéréotypée du commandeur ne tient qu'une place minime dans le texte, au vu de l'importance qu'il a eue dans la carrière du protagoniste (et malgré les 45 références à Jackson trouvées dans l'index).

La relative brièveté de l'ouvrage (188 pages) ne permettait peut-être pas une description épaisse du personnage. Pourtant, on reste un peu sur sa faim quant à la personne elle-même qui existe finalement assez peu, perdue dans le flot des événements et des actes politiques. Ce personnage complexe, à la fois secret et bon vivant, émerge peu en tant qu'homme dans la biographie ; on l'" entend » également peu puisque ce sont ses paroles politiques qui priment. L'homme privé est relativement absent, sauf peut-être au tout début (5-8) et à la fin (158-162), comme si le politique effaçait l'humain. C'est peut-être dans les descriptions de sa mondanité qu'il existe davantage (146). Bien sûr, c'est une biographie politique, mais l'homme n'affleure-t-il pas à chaque instant de la vie du politicien et ne doit-il ainsi pas trouver sa place dans l'exposé de toutes les identités qui forment celui que l'on nomme Martin Van Buren?

9 Pour reprendre l'opinion de Tom Coens sur la façon dont Joel Sibley traite du même sujet, on peut dire que Loizeau déploie parfois une «affection partisane » envers Van 
Buren. Durant la révision de la Constitution de l'État de New York, Van Buren est censé avoir joué un grand rôle et Loizeau loue "l'étendue impressionnante de son savoir juridique et la sagesse et la clairvoyance de ses positions ", mais il reconnaît un peu plus loin que bien des points du nouveau texte permirent ensuite de limoger les hommes de son adversaire politique. Sagesse juridique ou clairvoyance politique? Loizeau semble adhérer un peu facilement à ces pratiques ambiguës. De même, la création de la machine politique de New York, the Albany Regency, ancêtre du Tamany Hall de triste mémoire - ne suscite guère que des louanges sur le mode de l'excellence de l'organisation ou la discipline des troupes (38-39). Loizeau souligne l'intérêt de Van Buren pour la structure partisane (à laquelle s'opposait la génération des Pères Fondateurs), mais ne dit rien de l'emballement du système de la machine politique et de son boss qui pervertira le système politique local jusque dans les années 1950. Or, les racines du mal sont apparentes dès la constitution du système à la fin du XVIII ${ }^{\mathrm{e}}$ siècle.

Toutefois, le propos est clair et les efforts de contextualisation qui sont déployés sans cesse dans le livre éclairent le lecteur sur des questions parfois complexes, comme l'opposition des Bucktails à la faction du gouverneur Clinton DeWitt à New York (27-34) ou encore le projet de independent treasury pour combattre la dépression de 1837 (face aux circonstances exceptionnelles, Van Buren renversait «l'équilibre politique qu'il avait constamment tenté de maintenir » en déposant les fonds fédéraux dans des soustrésoreries et non dans les banques régionales en partie responsables de la crise [130]).

11 Enfin, Loizeau montre bien l'attachement viscéral de Van Buren à l'Union. La carrière nationale de ce président est à cet égard atypique, due en partie à sa longévité (il vit huit présidents différents lui succéder avant de mourir en 1862). En effet, son engagement dans la vie politique dépasse de loin sa période présidentielle et Loizeau précise qu'il travailla sans relâche après son mandat unique contre l'extension de l'esclavage dans les territoires de l'Ouest. Son action est ambiguë car il favorisa toujours le droit des États (et donc l'acceptation de l'esclavage dans les États sudistes) afin de préserver l'alliance Nord-Sud; lui-même était d'ailleurs issu d'une famille esclavagiste de New York (Loizeau rappelle très justement que cet État abolit l'esclavage en 1827 seulement). Durant ses années au pouvoir, il adopta une attitude d'obstruction face à l'abolitionnisme. Mais la progression de l'esclavage dans les États de l'Ouest, notamment le Texas, et surtout les conflits toujours plus nombreux dans les nouveaux territoires entre esclavagistes et Free Soilers, qui militaient activement contre l'institution particulière, le préoccupaient au plus haut point. Sa prudence refléta son désir constant mais impossible de maintenir les équilibres précaires de la république (135-136).

uren ne cesse de prouver comment, tout au long de sa carrière, ce que l'on nomme modération ou non-engagement était surtout lié au désir de trouver un compromis entre factions, entre régions, pour éviter le carnage d'une guerre civile. Et si Little Van est critiquable sur bien des points, il est assez admirable dans son obsessive attention à trouver les compromis, à éviter les humiliations, à organiser la démocratie, sans désavouer ni abandonner, tant s'en faut, le conflit, la lutte, la stratégie, le calcul. Van Buren nous donne en somme, à travers le texte de Loizeau, une leçon de démocratie, avec ses principes, mais aussi ses travers, ses intrigues, ses manigances, et ce qui ressort est une tentative de vivre selon ses croyances politiques et philosophiques sans se voiler la face sur les compromissions nécessaires qu'implique un système certes imparfait, mais dont Van Buren avait adopté les idéaux. Loin des figures 
héroïques du panthéon, Old Kinderhook mérite sans doute une place reconnue dans l'histoire américaine et la biographie efficace de Loizeau nous aide à en prendre toute la mesure.

INDEX

Thèmes : Recensions

AUTEUR

JEAN-MARC SERME

Université de Bretagne Occidentale 